\title{
The E-Method: a highly accurate technique for gene-expression analysis
}

\begin{abstract}
Roche Applied Science has repeatedly set standards for high-speed real-time PCR systems. The new LightCycler ${ }^{\circledast} 80$ System offers different methods of data analysis for relative quantification of geneexpression behavior. Whereas the $\Delta \Delta C_{T}$ Method provides fast, easy analysis of gene expression, the E-Method from Roche Applied Science can produce more accurate relative quantification data by compensating for differences in target and reference-gene amplification efficiency, either within an experiment or between experiments.
\end{abstract}

Twenty years ago, the PCR - an amazingly simple idea that would become one of the most revolutionary scientific techniques of the 20th century - was first described. Since then, the invention of realtime PCR technology has permitted further revolutionary advances in DNA analysis. For years, Roche Applied Science has set standards for innovative, high-speed real-time PCR systems.

The innovations started with the carousel-based LightCycler ${ }^{\circledR}$ RealTime PCR Systems (32 capillaries per run), which are currently widely accepted by the scientific community. Roche Applied Science now extends this tradition of innovation and high performance to higherthroughput applications with the new LightCycler 480 System (96and/or 384-well plate format).

The cutting-edge LightCycler 480 System (Fig. 1) design includes modular instrumentation and software, high-performance reagents and tailor-made disposables, all of which guarantee the best PCR results. Technological enhancements in the LightCycler 480 System, which include a sophisticated thermal cycler and optical system, permit rapid and precise analysis of gene expression and genetic variation. When combined with assays from Roche Applied Science's new Universal ProbeLibrary - a new probe-based, yet highly flexible, gene-expression assay system — the LightCycler 480 System provides convenient fast-tracking of many research activities.

Effect of efficiency on relative quantification methods Real-time PCR can be used to accurately quantify a target nucleic acid, using either absolute quantification methods (for example, to

\section{Gudrun Tellmann}

Roche Applied Sciences, Nonnenwald 2, 82372 Penzberg, Germany. Correspondence should be addressed to G.T. (gudrun.tellmann@roche.com).

PUBLISHED ONLINE 21 JUNE 2006; DOI:10.1038/NMETH894

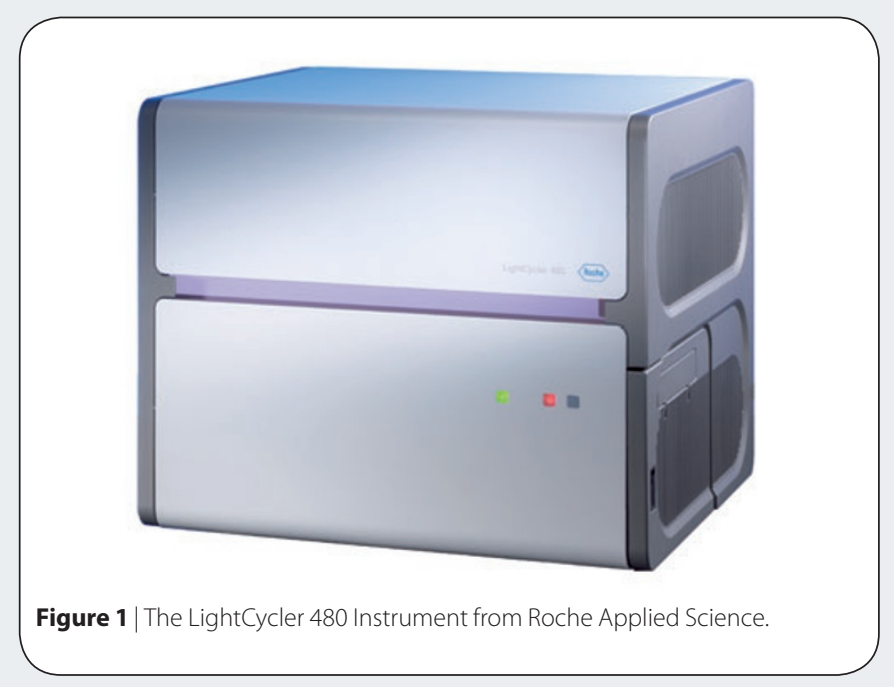

determine the actual number of viral copies in a sample) or relative quantification methods (for example, to determine the ratio of a regulated target gene to an unregulated housekeeping gene in the same sample $)^{1,2}$. As the name suggests, the latter method calculates the normalized values of one gene's expression level relative to another rather than absolute values.

The relative quantification method has become the method of choice for investigating changes in gene expression behavior, such as the expression of a tumor suppressor gene under non-disease and disease conditions. However, the reliability of all relative quantification calculations depends on the quality of the PCR.

Efficiency is a quantitative expression of the quality of the PCR process. The highest-quality PCRs run with an efficiency of 2, meaning that the number of target molecules doubles with every PCR cycle. Unfortunately, PCR efficiency is highly sensitive to many aspects of the reaction that vary from run-to-run (for example, sample preparation, 
nucleic acid purification, PCR primers and probes). Consequently, two different PCRs may not have identical efficiencies.

\section{A choice of relative quantification methods}

For precise relative quantification, the most recent generation of LightCycler Systems (the LightCycler ${ }^{\circledR} 2.0$ Instrument and the LightCycler ${ }^{\circledR} 480$ Instrument) offers a choice of different methods of data analysis, such as the $\Delta \Delta \mathrm{C}_{\mathrm{T}}$ Method and the E-Method, each of which handles the problem of efficiency differently (Fig. 2). Furthermore, these software modules allow fast-tracking research activities based on a highly flexible design.

The $\Delta \Delta \mathrm{C}_{\mathrm{T}}$ Method was the method used in early relative quantification experiments, and it can still provide fast, easy analysis of gene expression $^{3}$. This method, however, only provides reliable quantitative data if certain assumptions inherent in the method are met: the efficiency of the PCR assays for both target and reference genes must be optimal and/or identical. For the $\Delta \Delta \mathrm{C}_{\mathrm{T}}$ calculation, the efficiency of both PCRs is assumed to be 2 , which represents a doubling of molecules in each cycle. But if target and reference PCR efficiencies are neither identical nor optimal, the $\Delta \Delta \mathrm{C}_{\mathrm{T}}$ Method may produce incorrect gene expression data. In such cases, methods that are based on true efficiency values, such as Roche Applied Science's E-Method, can provide more accurate data.

The E-Method can produce more accurate relative quantification data because it can compensate for differences in target and reference gene amplification efficiency either within an experiment or between experiments. The E-Method analyzes the amplification efficiency of target and reference genes by using so-called relative standards. These standards are serial dilutions of a single sample (for example, undiluted, 1:10, 1:100 and so on), where concentration is expressed in relative units (for example, 1, 0.1, 0.01 and so on). By using such dilutions to generate a standard curve, the E-Method avoids the time-consuming preparation of artificial or cloned standards, and the determination of their absolute values. Additionally, as the relative standards contain normal sample material, their amplification efficiencies are very similar to those of all the unknown samples.

Furthermore, the E-Method can normalize for run-to-run differences, such as those caused by variations in reagent chemistry. For such normalization, one sample (for example, one of the relative standards) must be designated a calibrator for the target and for the reference gene. These calibrators are then used repeatedly in subsequent runs during the entire study to guarantee a common reference point and allow comparison of all experiments within the series.

\section{Conclusion}

The relative quantification software of the LightCycler Systems provides several highly accurate algorithms for relative quantification. Reliable gene-expression data can be generated with either the $\Delta \Delta \mathrm{C}_{\mathrm{T}}$ Method a

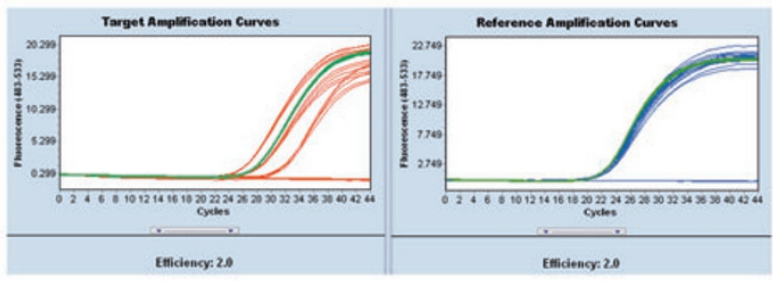

b

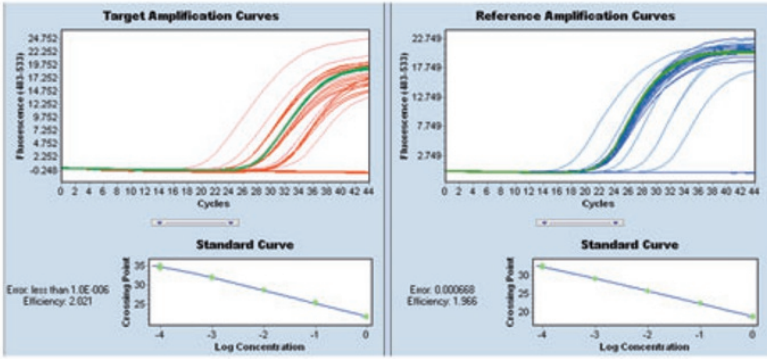

C

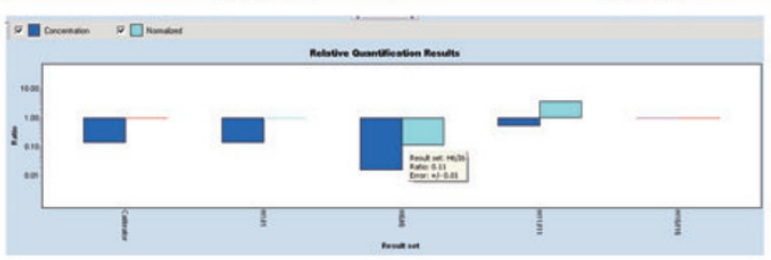

Figure 2 Two different relative quantification analyses of the same run. (a,b) A typical target and reference run, with unknowns (red and blue) and calibrator samples (green), is analyzed via either the $\Delta \Delta \mathrm{C}_{\mathrm{T}}$ Method (a) or the E-Method (b). Whereas the data in $\mathbf{a}$ are based on an assumption that efficiency $(E)=2$, the E-Method data are based on the true efficiency of each reaction, which is derived from serial dilutions of the target (light red; $E=2.021$ ) and reference genes (light blue; $E=1.966$ ). (c) The final results from $\mathbf{b}$ are automatically calculated from the crossing point (Cp) values of the target (unknowns and calibrator) and the reference (unknowns and calibrator).

or the Roche Applied Science E-Method, depending on the PCR efficiency of a particular experimental system.

Additional information about the LightCycler Systems and the described gene expression approaches is available on the Roche Applied Science Special Interest Page (http://www.lightcycler. com).

LIGHTCYCLER is a trademark of Roche. Patent and license disclaimer information is available online (http://www.lightcycler. com).

1. Edwards, K. et al. Real-Time PCR: An Essential Guide (Horizon Bioscience, Norfolk, 2004).

2. Bustin, S.A. $A-Z$ of Quantitative PCR (International University Line, La Jolla, 2006).

3. Livak, K.J. \& Schmittgen, T.D. Analysis of relative gene expression data using real-time quantitative PCR and the $2^{-\Delta \Delta C_{T}}$ method. Methods $25,402-408$ (2001).

This article was submitted to Nature Methods by a commercial organization and has not been peer reviewed. Nature Methods takes no responsibility for the accuracy or otherwise of the information provided. 